\title{
Editorial
}

\section{Some Initial Thoughts on Plasma Cosmology}

Jeremy Dunning-Davies*

Department of Physics, University of Hull, England

Some years ago, in an entirely different context, Sir Winston Churchill advised people to learn from the lessons of history. This advice might well be seen as applying to all fields of human activity and now, near the beginning of the 21 st century, it seems highly appropriate to follow this advice in several areas of physics which, at least in the eyes of some people, seem to be almost stagnating. Hence, in this spirit, it is of interest to note that, following the introduction of Newton's mechanical ideas, work still proceeded apace investigating electromagnetic phenomena and this continued at least into the earlier years of the twentieth century, as is evidenced by the contents of J. J. Thomson's book Electricity and Matter [1], which is concerned with a series of lectures he gave at Yale University in May, 1903. However, this book provides but one example to illustrate the very real emphasis on work involving the effects of the electric and magnetic fields, work which constantly sought an explanation in terms of those forces for the concept of mass. As an aside, it is possibly worth noting that, in this book, Thomson talks routinely of the equivalence of mass and energy, and that in lectures delivered in 1903. However, after those early years of the century, the emphasis seems to have shifted to explanations of phenomena purely in terms of gravitational effects as far as most mainline research has been concerned. Considering that it is accepted that much of the matter in the universe is in the form of plasma, this seems a retrograde step and this view is surely strengthened when the work of such as Kristian Birkeland and Hannes Alfvén is considered. One may only speculate as to why the emphasis of much scientific research changed in this way. However, thanks to people like Birkeland, Alfvén and (more recently) Peratt, work in the areas of electromagnetism and plasma physics did continue and it should be noted that much of the work on plasmas has been via laboratory experiments, so hard experimental evidence is available to support any claims made.

The work on plasmas and other electromagnetic phenomena has inspired some people to examine astronomical phenomena in these terms and this has resulted in the so-called Electric Universe idea as expounded, for example, in the books The Electric Universe by Wallace Thornhill and David Talbott [2] and The Electric Sky by

*Address correspondence to this autor at the Department of Physics, University of Hull, England; Tel: 01482650524;

E-mail: j.dunning-davies@hull.ac.uk
Donald Scott [3]. Reading through this material makes one immediately aware that, while like orthodox accepted theory, the electric universe ideas are supported by much computer modelling, it can also draw on parallels in astronomy with plasma phenomena observed in the laboratory. Admittedly, drawing such parallels involves scaling up tremendously but assuming this possible is little different from assuming that laws seemingly applicable here on the Earth are also applicable in the Solar System and, indeed, throughout the universe. However, at least visually, some of the phenomena observed in the laboratory are very like what is observed by some of the most powerful of telescopes; - electric currents in plasma naturally form filaments due to the so-called 'pinch effect' of the induced magnetic field. Electromagnetic interactions cause these filaments to rotate about one another to form a helical 'Birkeland Current' filament pair and this is very much the structure seen in the Double Helix nebula near the galactic centre; again, the Hubble image of the planetary nebula NGC6751 looks remarkably like the view down the barrel of a plasma focus device. Examples such as these prove nothing but should awaken people to the possibility of alternative explanations for astronomical phenomena.

Much of the laboratory work seems to have originated with the work of Kristian Birkeland more than one hundred years ago. It was during his Arctic expeditions at the end of the 19th century that the first magnetic field measurements were made of the Earth's polar regions. His findings also indicated the likelihood that the auroras were produced by charged particles originating in the Sun and guided by the Earth's magnetic field. Birkeland, though, was an experimentalist and is still known for his Terrella experiments carried out in a near vacuum and in which he used a magnetised metallic sphere to represent the Sun or a planet and subjected it to electrical discharges. By this means, he was able to produce scaled down auroral-type displays as well as analogues of other astronomical phenomena. These claims, however, were only vindicated finally by satellite measurements in the 1960's and 70's. To that point in time, his experimental and observational achievements had tended to be overshadowed by the purely theoretical predictions and explanations of the geophysicist, Sydney Chapman. Once again, powerful mathematics seems to have held sway over the more expected techniques of physics - experimentation and observation, with mathematics a mere tool to be used when necessary. This is not to decry Chapman's work but to emphasise the 
overwhelming importance of the physics when investigating natural phenomena.

Birkeland also showed experimentally that electric currents tend to flow along filaments shaped by current induced magnetic fields. Of course, this confirmed observations of Ampère that indicated that two parallel currents flowing in wires experience a long range attractive magnetic force that brings them closer together. However, as plasma currents come closer together, they are free to rotate about each other. Such action generates a short range repulsive magnetic force which keeps the filaments separated so that they are, in effect, insulated from each other and able to maintain their separate identities. The end effect is for them to appear like a twisted rope and it is this configuration which is termed a 'Birkeland current', as was mentioned earlier when the Double Helix nebula was noted as a possible example. Satellites orbiting above the auroras in the 60 's and 70's were able to detect a movement of ions, indicating that electric currents were present. Later missions found quasi-steady electric fields above the auroras following the magnetic field lines, thus lending some credence to Birkeland's claim of the existence of an electric circuit between the earth and the Sun. Some may be sceptical of this latter interpretation but it is undoubtedly true that much of the material in the universe is in the form of plasma and there is certainly electric and magnetic activity occurring in abundance. This means there are numerous very good reasons for considering the effects of the electromagnetic force in the universe, only one of which could be the resolution of the problem of the missing mass.

However, precisely what is the Electric Universe? In truth, it is really simply an hypothesis, a new way of interpreting known data by utilising both new and wellestablished knowledge relating to electricity and plasma. It should be emphasised immediately that, in this new interpretation, gravity still has a role to play but it is a secondary one since the electric force is so much more powerful. A major point to be stressed from the outset is that, in this interpretation of astronomical phenomena, scientists are able to call on evidence from laboratory based experiments to help form and support suggested explanations for a wide variety of phenomena. It has been found that, as explained in more detail in the above-mentioned books, an electrified plasma in a laboratory is a good model for providing possible explanations for many recently observed astronomical phenomena which, in several cases, have caused puzzlement for astronomers seeking explanations via more orthodox gravitationally based theories. This is not to say that gravity is ignored and regarded as irrelevant; rather, the possible effects of the electromagnetic force on astronomical phenomena are investigated while still recognising the importance of gravitational effects. In the electric universe, the gravitational systems of galaxies, stars, moons and planets are felt to have their origins in the proven ability of electricity to generate both structure and rotation in plasma. It is felt further that the force of gravity assumes importance only as the electromagnetic forces approach equilibrium. As has been noted already, great consternation has been caused in astronomical circles by the realisation that gravity, as presently understood, cannot explain much that is observed if the amount of mass available is as now felt to be present. Hence, instead of positing the existence of 'dark matter' or following the path of modifying Newton's well-tried law of gravitation significantly, it is suggested here that the possible effects of the electromagnetic force be examined to see if, in conjunction with orthodox ideas on gravity, these puzzling observations can be explained.

A point which is often relegated to the background when discussing the solution of problems through the introduction of dark matter is the fact that the missing mass, if there really is any missing mass, is not absent homogeneously throughout the universe; it is missing only in specific places - for example, in the outer regions of galaxies. Hence, possible solutions, such as the idea that neutrinos possess mass, which are essentially homogeneous in nature cannot be acceptable. It should be mentioned at this point though that, in the Electric Universe model, neutrinos do possess mass and are extremely important. They respond only weakly to massive objects such as stars and galaxies but form an extended atmosphere which, for example, refracts light around the Sun from distant stars and this offers an alternative explanation for the so-called gravitational bending of light. On the other hand, in this model, neutrinos are not required to explain galactic rotation although they must contribute to the masses of both stars and galaxies. Again, having some mass, neutrinos will not be distributed homogeneously.

However, returning to the realisation that much of the matter permeating the Universe is in the form of plasma, it might be remembered that these clouds of plasma respond to the well-known laws of Maxwell. Also, as pointed out by Scott in his book [3], another law, formulated by Lorentz, does help explain the galactic speeds alluded to earlier. This law states that a moving charged particle's momentum (speed or direction)can be changed by application of either an electric field or a magnetic field or both.

This seems a highly likely contributory factor, at least, causing galaxies to rotate as they are perceived to do but would indicate, contrary to the accepted view, that gravity has less to do with things than has been thought. However, it should be emphasised that nowhere is it being suggested that Newton's law of gravitation is wrong or in need of significant modification; it is simply being suggested that, in deep space where everything swims in a sea of plasma, the Maxwell - Lorentz electromagnetic forces dominate over those of gravity.

It might be remembered also that the Lorentz force alluded to here changes a charged particle's momentum and that change is directly proportional to the strength of the magnetic field through which the particle is moving. Further, the strength of a magnetic field produced by an electric current is inversely proportional to the distance from the current but the gravitational force between stars is inversely proportional to the square of the distance. This well-known difference between the two forces could lie at the heart of the problem of the galactic rotation curves; certainly it seems an avenue worth exploring further, especially considering the fact that more and more space missions are indicating that electromagnetic forces are distributed more widely throughout space and are, of course, many orders of magnitude stronger than gravitational forces. 
Much time, effort and money is spent worldwide on producing elaborate computer programs which purport to support the prevailing belief in the Big Bang as being the correct theory explaining how the universe originated. However, as well as a great many laboratory experiments being performed to help establish plasma properties [4] , it has been shown also, using the Maxwell and Lorentz equations, that streams of charged particles, such as are found in the intergalactic plasma, will evolve into the familiar galactic shapes under the influence of electromagnetic forces. The results fit extremely well with the observed velocity profiles in the galaxies and all this with no recourse to missing mass. Much of this simulation work has been carried out by Anthony Peratt and is reported in various issues of the IEEE Transactions on Plasma Science, a highly prestigious journal.

These short notes, although capable of standing alone, are meant to act as an introduction to the following articles devoted to an introduction to the ideas of plasma cosmology or, as it referred to by some, the electric universe. The first article by Dave Smith is meant to be a general overall view of the field and its general ideas. The other articles by Donald Scott, C. J. Ransom, and Wallace Thornhill give much more detail of some specific topics. It might be noted that, to some, the terms 'plasma cosmology' and 'electric universe' refer to somewhat different topics but here the terms are being used almost interchangeably and the whole purpose of this collection of articles is to draw the attention of a wider audience to the possible importance of electromagnetic effects in cosmology.

\section{REFERENCES}

[1] Thomson, JJ. Electricity and Matter. Westminster: Archibald Constable \& Co 1904.

[2] Thornhill, W.and Talbott, D. The Electric Universe. Portland: Mikamar Publishing 2002.

[3] Scott, DE. The Electric Sky. Portland: Mikamar Publishing 2006.

[4] Peratt, A. Physics of the Plasma Universe. New York, USA: Springer-Verlag 1992.

(C) Jeremy Dunning-Davies; Licensee Bentham Open.

This is an open access article licensed under the terms of the Creative Commons Attribution Non-Commercial License (http://creativecommons.org/ licenses/by-nc/3.0/) which permits unrestricted, non-commercial use, distribution and reproduction in any medium, provided the work is properly cited. 